\title{
Versuch einer Erklärung des Zusammenhanges zwischen Dolmen, Ma!- und Schalensteinen in Palästina.
}

\author{
Von Dr. Hans H. Spoer in Jerusalem.
}

Wir haben uns daran gewöhnt, von einem swastischen Kreis zu sprechen; vielleicht ist die Zeit nicht ferne, in welcher man von einem Schalensteinkreise redet. Es ist nicht meine Absicht, hier zu zeigen, welche Länder wohl in einen solchen Kreis' eingeschlossen werden müssen, noch, ob wirklich irgendwelche Verbindung besteht zwischen den Schalensteinen des nördlichen Europas ${ }^{x}$ und denjenigen in Palästina, oder ob dieselben unabhängige Gestaltungen religiöser und sozialer Ideen sind. Sondern ich beschränke mich hier nur auf einen Versuch, basiert auf mehrjähriges Studium dieser merkwürdigen Steinsymbole in Palästina, eine Erklärung zu finden für jene mysteriösen. Schalensteine, die jeder aufmerksame Beobachter an zahllosen Plätzen in Palästina findet.

Es ist notwendig zuerst festzustellen, an welchen Orten man solche Schalen antrifft:

I. in der Nähe alter Zisternen, Brunnen und Aushöhlungen in Felsen zur Aufbewahrung von Wein und Öl;

2. in Gegenden, die jetzt fast unzugänglich sind, auf Felsenwegen weit von menschlichen Wohnungen entfernt;

3. auf Dolmen;

4. auf Malsteinen;

5. an vertikalen Seiten der Felsen;

6. auf flachen Felsflächen z. B. in Gezer, Ta'annek und Megiddo.

Der Zweck der Schalen in Verbindung mit Zisternen und Brunnen ist augenscheinlich. Die grofen Schalen, - der Durchmesser mancher derselben ist beträchtlich - benutzte man damals, wie auch noch heute,

I Man vergleiche die interessante Zusammenstellung bei W. Jolly in den Proceedings of the Soc. Antiq. Scot. Vol. XVI, p. $300 \mathrm{ff}$, wo viele Schalensteine abgebildet und Literalurangaben über Abhandlungen in betreff der europäischen Schalensteine zu finden sind. 
als Tröge für das Vieh oder zum Waschen von Kleidungsstuicken, während die kleineren zum Aufrechthalten der Wasserkrüge dienten. Das letztere gilt auch ohne Zweifel von den Schalen, die sich um Öl- und Weinzisternen herum befinden. Diese Art Schale hat gewöhnlich einen dreieckigen Längsdurchschnitt zum besseren Festhalten der darein gestellten spitzbodigen Krüge.

Wie jedoch lassen sich die isolierten Schalen erklären, die weit ab von menschlichen Wohnungen in die flache Felsoberfläche eingehauen sind? Fragt man einen Eingeborenen, - und der würde eher ein Verbrechen begehen, als seine Unwissenheit einzugestehen, - was für eine Bewandtnis es mit den Schalen hat, so wird er antworten: atîka und dann erklären, daß es Wein- und Ölpressen seien, vorausgesetzt, daß die Schalen groß sind. Tatsache ist, daß diese Schalen, wenn sie sich in der Nähe einer Wohnung befinden, für solche und ähnliche Zwecke benutzt werden, und daraus schließt der Fellache, daß diese einsam stehenden Schalen wirklich für die angegebenen $Z$ wecke ursprünglich gemacht worden seien.

Es bedarf weiter keiner Beweisführung, um auch selbst den Skeptischesten davon zu überzeugen, daß diese Schalen, die oft nur ein paar Zentimeter tief sind, nicht zur Ansammlung von Regenwasser dienten, um das Vieh zu tränken, noch um darein Wasserkrüge zu stellen. Die Erklärung der Existenz solcher vereinsamt stehender Schalen nach einer utilitaristischen Theorie mu $@$ als verfehlt betrachtet werden, da selbst die größte Schale weder einen praktischen Wert als Öl- oder Weinpresse hat, noch genügend Wasser fabt, um als Trog dienen zu können. Die Erklärung muß daher anderswo gesucht werden.

Man findet auch, wie oben angegeben, Schalen auf Dolmen. In Palästina gibt es Dolmen, mit wenig Ausnahmen, fast nur auf der Ostseite des Jordans. Auf einer Reise nach dem Norden fand ich einen solchen auch auf dem westlichen Abhange des Tabors, nahe bei dem neuen Wege, ungefähr in $2 / 3$ Höhe.

Die palästinischen Dolmen bestehen aus drei, vier, fünf oder sechs Steinen, mit oder ohne Boden und Rücken; derjenige auf dem Berge Tabor ist ungefähr $1,25 \mathrm{~m}$ hoch, andere sind bis $\mathrm{zu} 2 \mathrm{~m}$ hoch. Die horizontale Platte ist manchmal über $4 \mathrm{~m}$ lang und gewöhnlich etwas schief aufgelegt. Das Material, aus welchem die Dolmen hergestellt werden, ist der Stein, der an Ort und Stelle gefunden wird, z. B. metaphorischer Kalkstein, oder ein feuersteinähnlicher. Stein, chert genannt.

... 18.9 .08 . 
Für gewöhnlich fehlen den Dolmen diese mysteriösen Schalen, tatsächlich sind nur wenige bekannt mit diesen schalenförmigen Vertiefungen und ich gebe hier eine Liste der Fundstätten solcher Dolmen, bei welchen kein Zweifel über künstliche Bearbeitung der Schalen obwalten kann.

I. Dolmen in der Nähe von 'Ammān عَّان:

I. Auf der Südseite der Stadt ist ein Dolmen mit einer Platte, die $4 \mathrm{~m} \mathrm{O}$ nach $\mathrm{W}$ und $3 \% \mathrm{~m} \mathrm{~S}$ nach $\mathrm{N}$ mißt. Auf der Platte sind verschiedene kleine, durch Kanäle verbundene Schalen, die größte in der Mitte mibt $66 \mathrm{~cm} \times 51 \mathrm{~cm}$ bei einer Tiefe von $30 \% / 2 \mathrm{~cm}$. Die Platte liegt, wie gewöhnlich, schief. ${ }^{1}$

2. Westlich von dem Burghügel befindet sich eine Gruppe von Dolmen. Einer derselben, mit einer Platte von 2,44 m im Geviert, hat mehrere kleine Schalen und an der westlichen Ecke eine Öffnung von $76 \mathrm{~cm} \times 30^{3} / 2 \mathrm{~cm}$ Breite und $7 \% / 2 \mathrm{~cm}$ Tiefe. ${ }^{2}$

II. Dolmen in el-ḳalūḥ التقوح:

3. Ein Trilithon mit einer Schale in der Platte an dem verjüngten Ende derselben. 3

4. Ein gefallener Trilithon mit sechs scharf eingeschnittenen Schalen in der Platte. Diese haben einen Durchmesser von je $25^{x} / 2,23,10,7^{x} / 2$, 5 und $5 \mathrm{~cm}$ und eine Tiefe variierend von $13 / 4$ bis $\mathrm{zu} 5 \mathrm{~cm}$.

5. Ein gefallener Trilithon mit einer Platte von $2,59 \mathrm{~m}$ Länge und $2 \mathrm{~m}$ Breite, in welcher sich drei Schalen befinden; zwei derselben berühren einander und haben einen Durchmesser von $18 \mathrm{~cm}$ bei einer Tiefe von $7 \% / 2 \mathrm{~cm}$. Die dritte Schale hat einen Durchmesser von $8 \mathrm{~cm}$ und eine Tiefe von $10 \mathrm{~cm} .5$.

6. Dolmen mit einer Platte von $1,37 \mathrm{~m} \times 1,68 \mathrm{~m}$. In dem flachen Felsen auf der Ostseite des Dolmen, ganz in der Nähe, befindet sich eine Schale. ${ }^{6}$

7. Die Platte dieses Dolmen ist ungewöhnlich dick, 1,531. $\times 1,32 \mathrm{~h}$. $\times 1,37 \mathrm{~m}$ breit. . Seine Schale befindet sich ganz in der Nähe in dem flachen Felsen und hat einen Durchmeṣser von $53 \% \mathrm{~cm} .7$

III. Dolmen in der Nähe von Heschbon: el-ḳurmîyeh القرميّه:

8: Dieser ist der beste aller uns bekannten Dolmen. Die Steine haben den Anschein, als wenn sie künstlich geglättet wären. Es ist ein Trilithon mit Bodenplatte. Die obere Platte ist $2,44 \mathrm{~m} \times 2,74 \mathrm{~m}$ lang.

I Quart. Stat.: PEF 1882, p 76; Survey of E. Pal. 1889, Vol. I, p. 20; Conder:

$\begin{array}{llll}\text { Heth and Mloab } 3 \text { p. } 252 . & 2 \text { Survey p. } 22 . & 3 \text { Survey p. } 126 . & 4 \text { id. p. } 128 . \\ \text { CONDER op. cit. p. } 256 . & 5 \text { Survey p. } 130 . & 6 \text { id. p. } 128 . & 7 \text { id. p. } 132 . \\ \text { Zeitschrift f. d. alttest. Wiss. Jahrg. } 28.1908 . & & \end{array}$


Längs zwci Seiten befinden sich sechs bis sicben Schalen, deren Durchmesser zwischen $30 \mathrm{~cm}$ bis $46 \mathrm{~cm}$ variieren. 3

9. Mehrere Dolmen mit Schalen in dem flachen Felsen vor dem Dolmen, ${ }^{2}$ wie bei denjenigen zu el-ḳalûh (s. Nr. 5).

IV. Dolmen in der Nähe von Șumîyeh موميه:

10. Die Platte dieses Dolmen ist $2,14 \mathrm{~m} \times 1,53 \mathrm{~m}$ und hat auf dem sich verjüngenden Ende eine Schale von einem Durchmesser von $15 \mathrm{~cm}$ bei einer Tiefe von $5 \mathrm{~cm} .{ }^{3}$

II. Dolmen, ohne Maßangabe, mit fünf Schalen von ro bis $25^{x} / 2 \mathrm{~cm}$ Tiefe in der Platte, die eine regelmäßigere Form hat, als man für gewöhnlich findet. 4

V. Dolmen im Wâdi ğideid وادىجديد:

12. Die Platte dieses Dolmen ist $3,04^{\mathrm{x}} / 2 \mathrm{~m} \times 2,59 \mathrm{~m}$, auf ihr sind zwei Schalen von $25 \% / 2 \mathrm{~cm}$ und $35 \% / 2 \mathrm{~cm}$. Durchmesser bei einer Tiefe von $15 \frac{1}{2} \mathrm{~cm} .3$

13. Zu Anfang des Tales ğideid am Rande der Belka befindet sich ein Dolmen mit einer Platte von $2,59 \mathrm{~cm} \times 1,69 \mathrm{~cm}$, welcher mehr als 40 Schalen aufweist. Die größte Schale hat einen Durchmesser von $25 \% / 2 \mathrm{~cm}$ und eine Tiefe von $23 \mathrm{~cm}$. Fünf Schalen befinden sich fast in der Mitte des Steines in einer geraden Linie und sind, wie jene zu'Ammân, durch einen Kanal verbunden, während die übrigen Schalen an den Rändern der Platte angebracht sind. Dieser Dolmen hat eine Hinterwand. ${ }^{6}$

14. Die Platte dieses Dolmen mißt $3,04^{\mathrm{T}} / 2 \mathrm{~m} \times 2,44 \mathrm{~m}$, hat fünf Schalen, von welchen vier je einen Durchmesser von $18 \mathrm{~cm}$ und eine Tiefe von $10 \mathrm{~cm}$.haben, während eine Schale in der Mitte der Platte einen Durchmesser von fast $45 \mathrm{~cm}$ bei einer Tiefe von $30 \mathrm{~cm}$ hat. 7

I5. Die Platte dieses Dolmen ist 2,44 $\mathrm{m} \times 2,29 \mathrm{~m}$; er hat eine Hinterwand. Die so gebildete Kammer ist $1,53 \mathrm{~m}$ hoch, 2,73 $\mathrm{m}$ lang und $\mathrm{I}, 08 \mathrm{~m}$ breit. Der Boden besteht aus einer Steinplatte, in welcher sich eine scharf eingeschnittene Schale befindet von $30 \mathrm{~cm}$ Durchmesser und $15 \mathrm{~cm}$ Tiefe. Die Steine scheinen bearbeitet zu sein. ${ }^{8}$

16. Die Platte dieses Dolmen hat eine Schale von unregelmäßiger Rundung $53^{\mathrm{x}} / 2 \mathrm{~cm} \times 84 \mathrm{~cm}$ und einer. Tiefe von $15 \mathrm{~cm} .9$.

17. Dieser Dolmen hat wie Nr. I5 eine Hinterwand. Die obere Platte ist zerbrochen. In der Bodenplatte befindet sich ein rundes Loch,

\footnotetext{
Survey p. 163. 2 id. p. 17 I. 3 id. pp. 226 f. 4 CONDRR op. cit. p. 256.5 Survey p. $254 . \quad 6$ id. pp. 268 f. 7 id. p. $269 . .8$ id. p. 270. Conder op. cit. p. 258. 9 Survey p. 271.
} 
dessen Rand zerstört ist, es hat einen Durchmesser von $25 \% \mathrm{~cm}$ und ist ebenso tief. ${ }^{\text {. }}$

18. Dolmen mit Platte von $3,96 \mathrm{~m} \times 3,04 \% 2 \mathrm{~m}$ Größe, in welcher sich mehrere Löcher von verschiedener Größe befinden. ${ }^{2}$

19. In der Nachbarschaft von Nr. 18 befindet sich noch ein anderer gut bearbeiteter Dolmen mit einer Platte von $3,04^{\mathrm{x}} / 2 \mathrm{~m} \times 2,75 \mathrm{~m}$, welche mehrere roh ausgehauene Löcher aufweist. 3

Dolmen NO. von Ruğm el-Meseiyik am Rande der Belka:

20. Die Platte dieses Dolmen ist $3,66 \mathrm{~m} \times 2,75 \mathrm{~m}$ und hat drei Schalen von 8-10 cm Durchmesser bei einer Tiefe von $5 \mathrm{~cm} .4$

Neben diesen Dolmen erwähnt SCHUMACHER 5 noch andere, die eine Depression in der Mitte haben, ${ }^{6}$ ohne daß dieselbe direkt eine Schale wäre. Vielleicht sind diese Steine gerade wegen ihrer eigentümlichen Natur zu Dolmensteinen ausgewählt worden.

Die Araber nennen die Dolmen bët el-ṛ̂ul; obgleich sie dieselben nicht als heilig betrachten, so erachten sie es jedoch sehr häufig für notwendig, sich gegen den bösen Einfluß der vermeintlichen Bewohner durch Errichtung von kleinen Steinhaufen in der Nähe der Dolmen 7 zu schützen.

Ihre tischähnliche Form legt den Gedanken nahe, daß sie einen Altar oder Opferstein darstellen, während die kastenähnliche Gestalt, besonders derer mit Steinboden, auf ein Grab hinzudeuten scheinen.

Der einfachste Altar war ein Felsblock, I Sam 14 30, auf den das Blut der Gottheit ausgegossen wurde. . Das Opfermahl, sowie das Verbrennen eines Teiles des Opfertieres gehören einer späteren Zeit an, in welcher eine mehr entwickelte Theologie zur Geltung gelangt war. Derselbe primitive Altar-Fels wird erwähnt in Jdc $62013_{1} 19^{8}$ und Gen 28 I8ff., an welcher Stelle מצבה מבה gelesen werden muß, denn wo die Gottheit erscheint, wird ihr geopfert und dazu braucht man einen Altar und keine mașṣêba. Aufjerdem scheint v. II eher auf einen flachen Stein hinzuweisen, da er ja als Kopfkissen diente, als auf einen langen, säulenähnlichen Stein, der wohl schwerlich solchem Zwecke dienen konnte, wenn man nach dem urteilen darf, was noch an mașșēboth, grob und klein, im Lande zu sehen ist.

Der primitive einsteinige Altar wurde erweitert durch die Hinzufügung anderer Steine, wie die Erzählung des Vertrags zwischen Laban

: Survey p. $272 . \quad 2$ id. p. $266 \mathrm{f}$.

5 ZDPV 1886. Across Jordan 1886. The Jaulan.

5 ZDPV 1886. Across Jordan 1886. The Jaulan. 6 Abila Pell
p. 173. 7 2. B. im Jordantal. PEF. Quart. Stat. 1882 p. 77 f.

Jde $6_{20}$ ff. und $13_{29}$ ff. sind wohl nur Varianten derselben antiken Erzählung.
3 id. p. 268.

4 id. p. 273.

6 Abila Pella and North 'Ajlun

8 Die Erzählungen $18 *$ 
und Jakob zcigt, Gen 314648 5r. Der ל ל ist der Altar, welcher bei diesem feicrlichen Vertrage errichtet wurde und in dessen Nähe man die Opfermahlzeit einnahm. Man mag in dcm d das Äquivalent des Dolmen sehen, welcher aus einem einfachen heiligen Stein durch Hinzufügen von anderen Steinen in einen erbauten Altar umgewandelt wurde. Wir können hieraus schlielen, daß die Jakob- und Laban-Erzählung in ihrer gegenwärtigen Form jünger ist als I Sam 1433 oder selbst Gen 28 18, welche Erzählung, trotzdem sie von später Hand überarbeitet ist, nichtsdestoweniger die alten $Z$ üge treu bewahrt hat, nämlich, daf in dem heiligen Stein ein Numen, el, residiert, eine Auffassung; welcher man im AT sonst nirgends begegnet und welche vielleicht schon verloren war, als die Handlung I Sam 1433 f. vorgenommen wurde.

Der aus mehreren Steinen bestehende Altar Gen 3146 ist das Produkt eines höher entwickelten Kultes. Das Opferblut wurde nicht mehr einfach über den flachen Opferstein ausgegossen, wobei vieles auf den Boden lief und vom Erdreich aufgesogen wurde, wodurch der Gottheit, die in dem Steine wohnte, ein Teil des Opfers gleichsam entzogen wurde. Es war vielleicht dieses der Grund, weshalb die alten Araber oft unter ihrem Götzensteine eine Höhle hatten, in welche das Blut des Opfers lief; ${ }^{\mathbf{I}}$ dasselbe war auch der Fall in Jerusalem, wo unter dem Altarfels sich eine Höhle zur Aufnahme des für die Gottheit bestimmten Opferblutes befindet. Es ist der Natur der Gottheit angemessener, daß sie trinkt, da Durst ein subtilerer Appetit ist als Hunger und flüssige Nahrung einem feiner organisierten Körper mehr angemessen ist. ${ }^{2}$

Der nächste Schritt in der Entwicklung dieses Gedankens führte zur. Verbrennung des Opfers, oder eines Teiles desselben, wodurch man glaubte, die grobe materielle Masse in eine verfeinerte, ätherische umzugestalten, so da $@$ sie als Mahlzeit der Gottheit dienen könnte. Bei den Hebräern wurde diese letzte Entwicklung der Opferidee in der Zeit des Deuteronomiums erreicht, in welcher das Gesetz entstand, da $b$ alle Opfer an einem bestimmten Orte dargebracht werden mußten, I2 5.

Der Dolmen ist der heilige Opferstein, welcher durch Unterstellung anderer Steine, Gen 28 18, der direkten gefahrbringenden Berührung mit dem Boden entzogen wurde. Die Gefahr lag einerseits darin, daß der heilige Stein selbst entheiligt wurde durch äußere Verunreinigung, die leicht stattfinden konnte, wenn der flache Stein auf dem Erdboden lag; andererseits war es gefährlich für den Menschen, welcher der Gegenwart

I Wellhausen, Arab. Heidentum 2 p. 103.

2 Odyssee Buch XI. 
des Numens in dem heiligen Steine nicht bewußt, durch irgendeine unbedachte Tat die Rache desselben herausforderte. Die Erhebung des heiligen Steines mag auch außerdem noch vorgenommen worden sein, um ihm eine Ehrenstellung unter den anderen Steinen zu geben.

Den Höhlen, deren schon Erwähnung getan wurde in Verbindung mit den arabischen Götzensteinen und der sachra in Jerusalem auf dem Tempelplatze, entspricht die charakteristische Schale, welche sich häufig vor dem Dolmen befindet ${ }^{x}$ und ebenso wie jene zur Aufnahme des Opferblutes bestimmt ist. Die Schalen vor den Dolmen liefern vielleicht die Erklärung für die Existenz so vieler einzelner Schalen, die man überall in Palästina, besonders auf der Westseite des Jordans findet. Die fast gänzliche Abwesenheit von Dolmen auf dieser Seite des Jordans ist bemerkenswert, besonders im Hinblick auf die vielen Dolmen auf der Ostseite. Für dieses Phänomen finden wir die Erklärung in der israelitischen Geschichte: in dem Befehl Dtn $122 \mathrm{ff}$, wonach alle Heiligtümer der Ureinwohner zerstört werden sollten, alle מובחות und alle, eine Verordnung, die König Josia, gemäß II Kön 22 f., zum Austrag brachte. Der Dolmen-Altar konnte, da er aus losen Steinen erbaut war, leicht zerstört werden, anders stand es aber mit den in den Felsen gehauenen Schalen, die vor den Dolmen angebracht waren. ${ }^{2}$ Da die Hauptsache, der heilige Opferstein, zerstört war, so hatten übrigens die Schalen keine weitere Bedeutung. Josias Macht erstreckte sich nicht über den Jordan hinaus, und da dort kein ikonoklastischer König gegen andere Kulte kämpfte, so blieben im Ostjordanlande die heiligen Opfersteine verschont.

Lassen sich der Gebrauch und die Existenz der einen Schäle vor dem Dolmen auf obige Weise erklären, dann entsteht die Frage: wie läbt sich die Vervielfältigung der Schale und ihre veränderte Position auf der Platte des Dolmen erklären?

Mir scheint die Schale unten vor dem Dolmen ein älterer Kultgegenstand $z u$ sein als die vielen Schalen auf der Platte, ebenso wie der Einsteinaltar älter ist, als der aus' mehreren Steinen errichtete AltarDolmen. Aus denselben Gründen, aus denen man den einfachen heiligen Stein hochstellte, hob man wohl auch seinen Adjunkt, die Schale, und brachte sie in der bequemsten und natürlichsten Stellung an, nämlich auf der Platte des בית אל

x cf. Nir. 7. Ebenso 'Ammān und Heschbon, Survey p. 17 I. Conder op. cit. p. 256.

2 So auch Conoer in Quart. Stat. PEF 1882 p. 77. 
Das Ausgicßen des Blutes ist die älteste Form eines flüssigen Opfers, wie Eccl 5015 beweist, wo der Wein, der als Libation ausgegossen wird, aipa oidepuijis genannt wird." Aus anderen Stellen des AT erfahren wir die Existenz von Trankopfern; David gießt das Wasser von dem Brunnen zu Bethlehem als eine Libation aus, II Sam 23 17. Die Weinlibation wurde bei den Israeliten an dem Fulie des Altars ausgegossen, Num 28 7. Das Trankopfer, unabhängig von dem Brandopfer, ist zweifellos älter als das komplexe Brand- und.Trankopfer, in welchem das letztere über das erstere gegossen wurde, wie das Einfache dem Komplexen vorhergeht. Wir mögen a priori annehmen, daß mit dem Gebrauch von Wein und Wasser zu Libationszwecken die Libation quantitativ größer wurde als beim Blutopfer und daß man daher auch eine größere Anzahl Schalen nötig hatte als bei letzterem, da anderenfalls zu viel auf den Erdboden geflossen wäre. Diesem augenscheinlichen Bedürfnisse müssen wir die vielen, oft großen Schalen auf der Platte der Dolmen zuschreiben. Wir mögen vielleicht in dieser neuen Erscheinung einen sozialen Fortschritt des Volkes erblicken, von einem ausschließlich Viehzucht treibenden Volke hat es sich zu einer Ackerbau treibenden und seßhaften Bevölkerung entwickelt. Der Charakter der Dolmen als Altäre wird ferner durch Namen bezeugt, die in der Úberlieferung noch heute fortleben, z. B. der ḩağ $r$ ed-dumm in Obergaliläa. ${ }^{2}$

Jedoch angesichts der Funde, die Dr. SchumaCher zu 'ain dakkar ${ }^{3}$ in Dolmen gemacht hat, haben die Dolmen auch anderen als nur sakralen Zwecken gedient. Die Einheimischen nennen dieses Dolmenfeld Kubûr be ni is ra êl $=$ Gräber der Kinder Israels. Hier fand ScHumaCHer in Dolmen Knochen, zwei bronzene Ringe und Holzasche. Diese Sachen scheinen auf den Grabcharakter der Dolmen hinzuweisen. Wenn dieses wahr ist, was angesichts der Funde kaum verneint werden kann, wie verhält es sich dann, so mögen wir uns wohl fragen, mit ihrer Eigenschaft als Altäre? Um diese Frage zu beantworten, müssen wir uns kurz vergegenwärtigen, in welchem Verhältnisse sich der alte Semite zu seinem Vorfahren dachte.

Blutverwandtschaft zwischen Gottheit und Verehrer ist ein tiefgewurzeltes Prinzip in der semitischen Religion. ${ }^{4}$ Viele arabische Stämme

I In gewissen arabischén' Kultgebräuchen wurde Fruchtsaft als Surrogat für Blut benutzt. W. Robertson Smith, Kinship p. 26I f. J. Wellhausen, Heidentum 3 p. $125 \mathrm{ff}$.

2 Quart. Stat. PEF. 1882 p. 76.

3 ZDPV 1886 p. 267 ff. Separatabdruck p. 104. The Jaulân. p. 125 . Pella etc. p. 176 etc.

W. R. SMITH, Religion of the Semites 2 p. 289. 
benennen sich nach dem Namen der Gottheit, von welcher sie ihre Abstammung herleiten, z. B. „Söhne des Bedr" (= Vollmondes), „Söhne des Hilâl" (= Neumondes), "Söhne des Schems" ( - Sonne). Der Hauptanspruch, den der antike Semite machte, war leiblicher Nachkomme seiner Gottheit zu sein. Das königliche Haus von Tyrus und die edelsten Familien von Karthago machten den Anspruch, direkte Nachkommen des tyrischen Baals zu sein. ${ }^{x}$ Der Anspruch assyrischer und ägyptischer Könige, auf Gottessohnschaft ist zur Genüge in den Inschriften erwähnt. Aber auch das AT hat uns manche Spuren dieses alten Glaubens bewahrt in den Stammnamen 7 אשי רן, welches Namen von Gottheiten sind, die ursprünglich als die Ahnen dieser Stämme gedacht waren. In Num 2I 29 werden die Moabiter Söhne und Töchter des Kemosch genannt, während in Mal 2 II eine Frau die Tochter eines fremden Gottes genannt wird, ein Gedanke, der schon dem Propheten Hosea geläufig war, vgl. 57 . Obgleich die Stelle in Maleachi sehr spät ist, so beweist sie doch, daß der Schreiber Zustände kannte, in welchen Völker oder

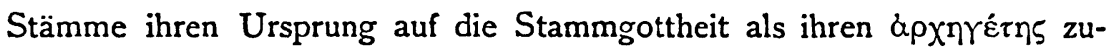
rückführten. Der Gott-Ahnenkult war dem alten Israeliten ebenso geläufig wie seinen Nachbarn; ${ }^{2}$ denn bis tief in die makkabäische Zeit hinein finden wir Spuren dieses Kultes, und es ist daher anzunehmen, dal die einwandernden Hebräer die ihnen verwandten Kulte mit der Übernahme der Heiligtümer auch übernommen haben.

Jedoch müssen wir noch einen Schritt weiter gehen und feststellen, ob Ahnenkult im engeren Sinne auch in Israel geübt wurde.

Die Patriarchen werden wohl niemals als ursprüngliche Gottheiten gedacht, sondern von jeher als Heroen, Götterlieblinge. Jeder der Patriarchen ist mit einem bestimmten Orte verbunden, Abraham mit Hebron, Isaak mit Beerscheba', Jacob mit Bethel und Joseph mit Schechem.

In der Abrahamsgeschichte haben wir vielleicht eine Verschmelzung zweier ursprünglich unabhängiger Kulte, nämlich: den heiligen Baum אלון mit seinem Numen, welches ursprünglich hier verehrt wurde, und die spätere Identifikation dieses Numens mit Abraham, dessen Kult von den Israeliten hier geübt wurde, bis er durch den Jahwismus zur Zeit Josias verdrängt wurde.

In Beerscheba; dem Hauptsitze des Heros Isaak, finden wir auch diese Verschmelzung zweier Kulte, des ursprünglichen Brunnennumens

2 Virgil, Aen. I. 729. Sil. Italicus I. 87.

2 Man vergleiche hierzu die interessanten Ausführungen Schwally's: Das Leben nach dem Tode. 
mit dem später aufgepflanzten Isaakkult; auch dieser verschwand erst zur Zeit Josias.

In Schechem, ähnlich wie in Hebron, haben wir den Baumkult, Gen 126 Dtn II 30 Gen 354 usw. verschmolzen mit dem des Joseph, Jos 2430 .

In Bethel finden wir dasselbe Phänomen, das lokale Numen ver-

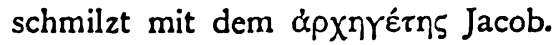

Es wäre verfehlt, anzunehmen, daß diese Patriarchen ursprünglich kanaanitische Gottheiten und als solche einfach von den Israeliten adoptiert waren; man würde bei dieser Annahme die Israeliten nicht allein ihrer Ahnen berauben, sondern auch von der Voraussetzung ausgehen, $\mathrm{da} ß$ sie als einziges unter allen Völkern niemals Heroenlegenden besessen hätten. Es muß jedoch zugegeben werden, daß Abraham usw. die Personifizierung zweier Kultideen ist; ebenso mub man nicht aus dem Auge verlieren, daß der Kultort älter ist als der Kult; vgl. Gen 2817 f.

Der Kult der Stammheroen führte nach und nach zu einem Kult des Hauptes der einzelnen Familien, mithin zu einem allgemeinen Ahnenkulte, wie man ihn klar dargestellt findet in den Trauergebräuchen und den Handlungen am Grabe (vgl. Schwally, op. cit.).

Der antike Araber versorgte seine Toten mit solchen Gegenständen, deren sie seiner Meinung nach bedurften. ${ }^{x}$ Der Dahingeschiedene verwandelte sich sofort in ein Wesen höherer Art, dessen Gunst durch Libationen von Wein und Wasser, die auf dem Grabe dargebracht wurden, gewonnen werden mußte. ${ }^{2}$ Hieraus erklärt sich, daß Grab und Heiligtum den Arabern verwandte Begriffe waren, ${ }^{3}$ welche Ansicht noch heute in der Veneration der Welis Ausdruck findet, da ihnen quasi die Stellung von Gottheiten eingeräumt wird, an deren Heiligtümern, Gräbern, Opfer dargebracht werden müssen.4 Ganz. dieselben Begriffe finden wir im AT. Der Israelit muß schwören, daß, wenn er Jahwe den Zehnten bringt, er nichts davon den Toten gegeben hat, $D \operatorname{tn} 26$ 14. Das von Jer 167 beschriebene Totenfest, welches niemehr stattfinden sollte, ist ein Opfermahl, woran der Tote teilnimmt.5 Dieses wird bestätigt durch Hos 94 , wonach keiner, der von dem Brote der Trauernden gegessen hat, vor Jahwe treten darf, weil er an einem anderen Kulte teilgenommen und sich dadurch verunreinigt hat. 6 Das Abschneiden der Haare - Jer 166 Am 8 10 Hes 7 18 und die Verbote in Lev 1927 Dtn $14 \mathrm{r}$ - war auch ein Toten-

I Wellhausen, op. cit. 2 p. 185.

3 W. R. SMITH, op. cit. p. $156 \mathrm{f}$.

5 W. R. SMITH, op. cit. p. 322 f.
2 id. p. $16 \mathrm{r}$.

4 Spoer, JAOS Bd. XXV (I.g04) p. 312 f.

6 STADE, Geschichte I. p. 389. 
Hans H. Spoer, Zusammenhang zw. Dolmen, Mal- u. Schalensteinen. 28I

opfer, wie es auch noch heute von einigen arabischen Stämmen dargebracht wird. ${ }^{x}$ Solche Opfer haben aber nur Sinn, wenn damit der Begriff des Fortbestandes des Dahingeschiedenen auf irgendeine Weise verbunden ist.

Wie Joseph unter dem heiligen Baume zu Schechem begraben und

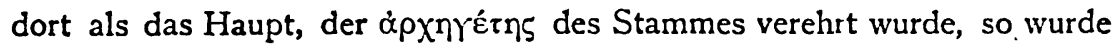
bald dem Haupte jeder einzelnen Familie nach dem Tode göttliche Verehrung dargebracht, jedes Familiengrab wurde zur Kultstätte. Gleichwie der heilige, auf der Erde liegende Opferstein zum Dolmen wurde, so wurde das Grab notwendigerweise zum Dolmen, d. h. zu einem heiligen Opferstein. Wir haben daher mit zwei Arten Dolmen zu tun, erstens dem ursprünglichen Opferstein, zweitens dem Opferstein-Grab, welches aus ersterem sich entwickelt hat, und auf beiden finden wir, gemäß dem Charakter dieser Steine, Schalen.

Man findet bei vielen in den Felsen gehauenen Steingräbern, die aus einer viel späteren Zeit stammen als die Dolmen, in der offnen Vorhalle oder zur Seite auf dem flachen Felsen, Schalen, und wir haben hier wohl eine der Zeit angemessene Entwicklung des Dolmen, des Opfersteingrabes.

Wir müssen noch eine vierte Art Schale betrachten, nämlich die, welche sich auf Malsteinen findet. Da diese Art Schale sehr selten ist, so gebe ich im folgenden eine Liste aller Malsteine mit Schalen, so weit ich imstande war, ihr Vorhandensein zu konstatieren.

\section{Bei 'Ammân:}

Mals teine.

I. I,37 $\mathrm{m}$ hoch mit einer Schale oben, die einen Durchmesser von $15 \frac{\mathrm{x}}{2} \mathrm{~cm}$. und eine Tiefe von $10 \mathrm{~cm}$ hat. ${ }^{2}$

2. Nordöstlich von der Zitadelle in der Ruine hadâdeh befindet sich ein umgestürzter Malstein von 4,27 m Länge, $1,98 \mathrm{~m}$ Breite und 6I $\mathrm{cm}$ Dicke mit einer Schale in der Seite von, $23 \mathrm{~cm} \times 13 \mathrm{~cm}$ Breite und $23 \mathrm{~cm}$ Tiefe.

II. Bei Nabiûs:

3. Bei dem sogenannten Grabe Josephs befinden sich zwei Malsteine, wovon jeder oben eine Schale hat.

III. Bei Arcot:

4. Die Malsteine, die um den Grabdolmen herum stehen, haben oben Schalen.

s Siehe die Nachweise bei W. R. SMith, op. cit. p. 324 Anm. 2.

2 Survey p. 23; Conder, op. cit. p. 253. 3 Survey p. 24.

4 Conder, op. cit. p. 238. 
IV. Bei El-mareiğât:

5. Dieser Malstein heißt bei der Bevölkerung hă y̆r el-manzûb und ist der künstlichste aller bekannten Malsteine (s. die Abbildung). Die

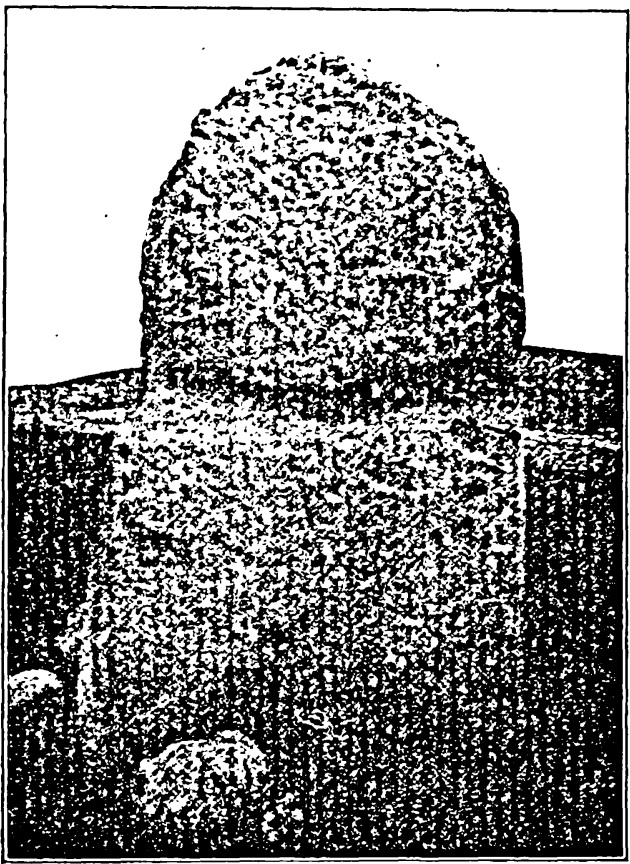
Spitze ist abgerundet, unterhalb derselben befindet sich eine Rinne von $15 \% / 2 \mathrm{~cm}$ (nach dem Survey $23 \mathrm{~cm}$ ) Breite bei $4 \mathrm{~cm}$ Tiefe. Die Rinne läuft halb um den Stein herum und ist ungefähr I $m$ hoch über dem Boden. Unterhalb derselben finden sich nach ScHICK ${ }^{x}$ zwei Schalen,während es nach dem Survey ${ }^{2}$ drei sind.

IV. Beim Tell el-matabae:

6. Ein umgestürzter $3,97 \mathrm{~m}$ langer und $1,53 \mathrm{~m}$ breiter Malstein mit einem unregelmäbig geformten Loche zwischen $20 \mathrm{~cm} \times 46 \mathrm{~cm}$ breit und $15 \mathrm{~cm}$ tief. 3

V. Auf der Halbinsel Sinai: 4

7. Nicht weit von der Wasserscheide, die von der Ebene von Errahâh gebildet wird, zur Rechten des Weges nach dem Kloster des Nağb Hawa befindet sich ein umgestürzter Malstein mit Schalen.

8. Ein anderer Malstein mit Schalen wird jetzt von den Beduinen als Grenzstein benutzt.

VI. Bei Gezer: 5

9. Malstein III hat eine Schale auf der westlichen Seite.

10. Malstein VIII hat zwei Schalen auf der westlichen Seite.

Ein Malstein hat eine schmale Rinne wie derjenige von El-mareiğât. VII. Bei Megiddo: ${ }^{6}$

12. Dort hat man einen schon bearbeiteten vierseitigen Malstein von .2,30 m Länge gefunden. Oben besitzt er eine Schale von $20 \mathrm{~cm}$ Durch-

Z ZDPV 1879 Karte II. 7.

2 Conder, op. cit. p. 26o; Survey p. 185 f.

3 id. p. 233. 4 Ordinance Survey of the Peninsula of Sinai.

5 Quart. Satt. PEF 1903 p. 28 f.

6 MNDP 1904 P. $47 \mathrm{ff}$. 
messer und einer Tiefe von $3-5 \mathrm{~cm}$ und auf der dem Osten zugewandten Seite noch eine zweite Schale von ungefähr II cm Durchmesser und $8 \mathrm{~cm}$ Tiefe.

13. Einen Kilometer nordöstlich von Megiddo, bei der chirbet elchazni, ist ein Malstein zutage gefördert worden von 2,25 $\mathrm{m}$ Länge mit Schalen am oberen Ende. ${ }^{x}$

14. Am nordöstlichen Fuße des Tells bei der chirbet 'ain el-kubbi ist ein Malstein mit mehreren Schalen, welche auf der Seite angebracht sind, gefunden worden. ${ }^{2}$

VIII. Bei Ta'annek: 3

15. Hier wurden zwei Malsteine gefunden, der eine mit der Schale oben und der andere mit der Schale auf der Seite.

- Der Malstein heißt im Arabischen نُصُب bekannt. Durch die Ausgrabungen ist erwiesen worden, daß der Malstein ein Kultobjekt in den Heiligtümern der vor-israelitischen Bevölkerung Palästinas war, auch ist er als solcher für die älteste israelitische Geschichte erwiesen, z. B. Hos 34 IO I 2, wo seine Zerstörung als eine Strafe von Jahwe für Israel in Aussicht gestellt wird. Die ältesten erwähnten Malsteine sind in Sichem Jos 24 26, Mizpa I Sam 7 12, Gibeon II Sam 208 , bei Gilgal gab es einen Kreis von zwölf Steinen Jos 4 5. Das Denkmal, welches in Gen 3 I 45 ff. beschrieben ist, ist nicht eine מצבה, sondern ein עומע, s. oben S. $275 \mathrm{f}$.

Die Frage ist nun, welche Stelle nahm die maṣṣ̂ba in dem ursemitischen Kulte ein?

Wenn man bedenkt, daß die Anfänge der semitischen Religion auf geschlechtliche Verbindungen zurückgehen, ${ }^{4}$ so muß man sich auch fragen, ob in dem Kultorte irgendeine sichtbare Repräsentation des Organs der Fruchtbarkeit dargestellt war.

Die alten Araber hatten eine männliche Gottheit, 'allâh, die durch einen aufrechtstehenden Stein symbolisiert wurde, nuṣb, während die weibliche Gottheit, 'allât, durch einẹn kegelförmigen Stein dargestellt wurde. Die lange und schlanke Form des einen Steines, oft spitzig oder nach obenhin sich verjüngend, läßt keinen $Z$ weifel aufkommen über das, was unter dieser Form dargestellt wurde, besonders im Hinblick auf das, was wir über die Anfänge der semitischen Religion wissen. Die Klassiker berichten, daß der Phallus durch eine Säule symbolisiert wurde; so standen in den Propyläen des Tempels von Hierapolis zwei Säulen, von

\footnotetext{
2 MNDP 1905 p. 3. 2 id.

3 E. Sellin, Tell Táannek 1904 p. 104.
}

4 Barton, Semitic Origins p. $106 \mathrm{f}$

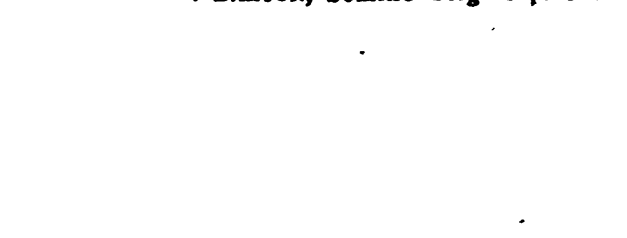


denen LUCIAN ${ }^{x}$ sagt, dal diese Phalli dem Dionysius und seiner Mutter Hera aufgestellt seien.

Infolge des Baus von Städten und anderen befestigten Plätzen wurde die במה mit ihren Kultgegenständen auch mit in den Kreis der Stadtmauern hineingezogen, respektive dort von neuem errichtet; denn das Heiligtum mußte mitten unter dem Volke bleiben, da sonst die Stadt ohne Gottheit wäre. Man findet aus diesem Grunde so viele mașsebôth in den jüngst ausgegrabenen Städten Palästinas. Aus demselben Grunde wurden. vor dem Tempel in Jerusalem zwei Säulen errichtet, die alten ehrwürdigen Kultsymbole, ohne welche eine במה nicht denkbar war und welche seit alter Zeit auf den במות Schechem, Gilgal, Mizpa und anderen mehr verehrt worden waren. Es ist wohl kaum zu bezweifeln, daß.der nuṣb oder die mașệba als Phallus das Symbol der semitischen Gottheit wurde ${ }^{2}$ und als solches sowohl Jahwe wie auch die anderen semitischen Gottheiten darstellte und von den Israeliten verehrt wurde bis in das Zeitalter des Josias. 3

Das AT bietet jedoch noch einige andere Beispiele, welche dartun, $\mathrm{da} \cap$ der Phallusdienst eine hervorragende Stelle in der Religion der Hebräer einnahm; diese sind: der Schwur Eliezers תחת ירך Abrahams, Gen 24 2; Josephs Schwur תחת ירק Israels, Gen 47 29. In diesen Stellen kann doch nur auf das Zeugungsglied hingewiesen werden wollen, wie dies ersichtlich ist durch einen Vergleich dieser Stellen mit dem Ausdruck

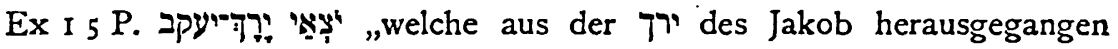
waren", vgl. auch Gen 4626 P, Jdc 8 30. Das . Wort ירך ist in diesen Stellen in einem ganz speziellen Sinn gebraucht, es bezeichnet hier den Sitz oder das Organ, aus dem die Nachkommenschaft des Mannes hervorgegangen ist. Die Schwüre selbst waren von ungeheuerer Wichtigkeit, hing doch z. B. von der Ausführung desselben in Gen 2429 die Nachkommenschaft Abrahams ab und alles was dieses für den antiken Menschen in sich schloß. Aus diesem Grunde hat die Tradition auch die Eideszeremonie treu bewahrt, welche selbst die späteren priesterlichen Schreiber nicht $z u$ unterdrücken oder auch nur $z u$ ändern wagten, da sie sich doch wohl völlig des hohen Ansehens bewubt waren, in welchem die Volksreligion ihrer eigenen Zeit einen solchen Schwur hielt. - Ein neues Element, wichtiger. als alles andere, findet sich in dem Umstande,

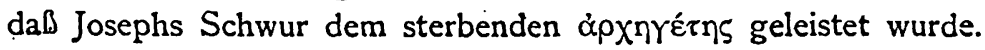

I Dea Syr. XVI. · 2 BARToN op. cit. p. 102.

3 id. p. 290; vgl. II Reg $233_{4}$ Dtn 75 . 
Als letztes Moment haben wir dann noch den Befehl in Ex 2842 zu erwähnen, wonach alle Priester Hosen tragen müssen, besonders wenn sie zum Altar Jahwes kommen. Da, wie Holzinger annimmt, die die Entblößung des Priesters ausschließt, so ist dieses Gesetz erst recht merkwürdig und kann nur verstanden werden im Hinblick auf einen in Israel wohlbekannten Phalluskult, womit das neue Gesetz auf jeden Fall brechen wollte, und daher wurden auch einschneidende Verordnungen in betreff der Kleidung der Jahwepriester gegeben. Das Symbol jenes Kults war von nun an mit Opprobrium bedeckt und daher finden wir diese Vorschrift in bezug. auf die Bekleidung jenes Teiles des Körpers bei den Priestern.

In Verbindung mit dem Vorhergehenden möchte ich auf einen Gebrauch hinweisen, der im nördlichen Syrien zu Hause ist. Die muslimischen Bewohner von Hamath schwören beim Phallus Gottes, während in Bludan, einem Orte ungefähr $40 \mathrm{~km}$ westlich von Damascus, selbst die einheimischen Christen dasselbe tun. ${ }^{x}$

Für die Annahme, daß der Malstein das Symbol des Phallus ist, haben wir erstens den allgemeinen archäologischen Beweis und zweitens die Literaturangaben, wonach dieser Symbolismus auch in und um Palästina in Gebrauch war. Im folgenden soll nun das Verhältnis der Malsteine zu den Schalensteinen untersucht werden.

Kegelförmige Steine, womit die alten Araber ihre weibliche Gottheit bezeichneten, finden sich auch nach .CONDER ${ }^{2}$ bei El-mareiğât und Minjeh und sind von ihm mit dem Steine der Allât identifiziert worden. Diese kegelförmigen Steine haben niemals Schalen, da, wie CoNDER sagt, 3 nur der. männlichen Gottheit blutige Opfer gebracht wurden. Dieser Schluf scheint mir jedoch nicht ganz zutreffend, da auch andere Arten Opfer oder Libationen einer Schale bedurften, wie auch dadurch erwiesen wird, dab bisweilen, nach ConDERs eigner Angabe, sich ganz in der Nähe dieser Steine solche Schalen befinden. 4

Der Phalluskult spielte, wie wir oben sahen, in antiker Zeit eine hervorragende Rolle im religiösen Leben der alten Semiten und er verdankte seine Wichtigkeit wohl solchen sozialen Verhältnissen, die dem Nanne oder Vater eine ganz unvergleichlich hervorragende Stellung dem Weibe oder der Mutter gegenüber einräumten. Die alten Araber stellten die männliche sowohl wie die weibliche Gottheit durch Steine, die eine bestimmte Form hatten, symbolisch dar. $\mathrm{Da}$ nun das eine

\footnotetext{
I Curtiss, Prim. Semitic Religion pp. $112 \mathrm{f}$.
}

2 Quart. Stat. PEF. 1882 
Symbol das männliche Glied darstellte, so liegt es nahe, in dem anderen Steine an ein Symbol der weiblichen Gottheit, entsprechend demjenigen der männlichen Goltheit, zu denken, sei es nun das weibliche Organ oder die weibliche, umgekehrt dargestellte Brust als die Quelle des Lebens für das neugeborene Kind.

Wir haben jedoch oben gesehen, dab es Malsteine gibt, welche an den Seiten Schalen haben. Diese Schalen, an den vertikalen Seiten des Malsteins, waren wohl kaum, wie gewöhnlich angenommen wird, zur Aufnahme von dickflüssigen oder fast festen Opfergaben, wie Honig, Butter oder dergleichen, bestimmt. Es würden ja wegen der grolen Hitze solche Opfergaben gar nicht in der Schale bleiben, sondern bald auslaufen. Nur die Schalen oben auf den Malsteinen waren wohl zur Aufnahme von Libationen bestimmt. Nach CoNDERs Angaben und so weit wie ich imstande war, der Sache nachzugehen, gibt es nun Schalen nur in Verbindung mit Malsteinen und nicht mit den kegelförmigen Steinen, den Symbolen der weiblichen Gottheit. Diese letzteren aber sind auch niemals in Verbindung mit Malsteinen, auf denen Schalen sind, gefunden worden und wir sind daher wohl $\mathrm{zu}$ dem Schlusse berechtigt, dab die Schalen an den Seiten der Malsteine ein Substitut für die kegelförmigen, die weibliche Gottheit darstellenden Steine sind, mithin selbst das darstellen, was jene Steine symbolisieren, vielleicht die weibliche Brust. ${ }^{x}$ Dieses stimmt, wie oben schon gezeigt wurde, ganz mit der ursemitischen Idee des Göttlichen überein und mochte deshalb auch mit vollem Rechte symbolisch dargestellt werden.

Die hier ausgesprochene Ansicht findet eine Bestätigung in HERODOT ${ }^{2}$, welcher sagt, daß er in Syrien Stelen gesehen habe, worauf ruvoukòs aiooĩ eingemeißelt waren, d. h. Malsteine, Symbole des männlichen Zeugungsgliedes, worauf das Symbol des weiblichen angebracht war. In anderen Worten, genau das, was durch die Malsteine mit Schalen dargestellt wird, die er wohl damit gemeint hat! Die Vereinigung dieser beiden Symbole repräsentiert eine bestimmte Entwicklungsstufe des sozialen Lebens der alten Semiten. Es ist nicht die des Patriarchats, in welcher das Weib, die Mutter, quasi rechtlos war, noch die des Matriarchats, in welchem la recherche de paternité unmöglich war, sondern es war die Stufe, in welcher die Zusammengehörigkeit des Mannes und Weibes als Häupter einer Familie fest begründet war. Und wie konnte dieser

I Ich erinnere in Verbindung hiermit an die Milchgrotte zu Bethlehem, wobin noch heute viele Katholikinnen wallfahren. Vgl. auch den Ursprung der Milchstrabe in der griechischen Mythologie.

2 op. cit. II. 106. 
Fortschritt besser dargestellt werden als dadurch, daf man dasselbe auch für die Gottheiten annahm und dieses durch eine Vereinigung der beiden göttlichen Symbole ausdrückte! In Verbindung hiermit weise ich auf die androgyne Gottheit hin, die einer Zeit angehört, in der die Unschuld dieses Kultes schon längst verschwunden war und die tierischen Lüste des. Menschen das in den Schmutz gezogen hatten, was seinem Ursprunge nach wahrhaft göttlich ist, denn wie Prof. BARTON ${ }^{x}$ mit vollem Rechte sagt: "The Semite actually hit upon a feature of human life which is, as scientific investigation is showing us, intimately connected with religious feeling at the present day, and has had more real influence in developing moral, altruistic and humanitarian feeling in the past than any other."

Es bleibt jetzt noch übrig zu untersuchen, welches die Bedeutung solcher Schalen ist, die alleine vorkommen, und solcher, die sich an vertikalen Felswänden befinden.

Schon zu Anfang wies ich auf den Gebrauch und Ursprung mancher Schalen hin; diese kommen hier nicht mehr in Betracht. Es gibt wenige Schalen, die sich an vertikalen Felswänden befinden, tatsächlich habe ich nur eine einzige Stelle angeführt gefunden. ${ }^{2}$ Hiernach befinden sich zwei Schalen bei Tell eṣ-șâfi, und ich habe selbst mehrere gesehen zwischen Bêt-ğebrìn und Tell el-ḥesi, in einem Distrikte nicht weit entfernt von Tell eṣ-șâfi. Diese Schalen können aus demselben Grunde wie jene, die in Verbindung mit Malsteinen sind, keine Opferschalen sein. Was sind sie? Es gibt ein kleines Modell eines phönizischen Tempels ${ }^{3}$ aus Terrakotta, welches zwölf runde Löcher in der Vorder- und einer Seitenmauer hat. In dem To:weg steht ein anthropoidischer Vogel, aus jedem der Seitenfenster sieht eine Frau heraus. Der menschenähnliche Vogel ist wohl eine Repräsentation der Astarte, welcher die Taube heilig war, auf jeden Fall aber scheinen die beiden Frauen darauf hinzuweisen, daß wir es mit einem Kultorte einer weiblichen Gottheit zu tun haben. Nachdem wir geșehen haben, was die Schalen in Verbindung mit dem Malstein-Phallus darstellen, wird die Annahme nicht unberechtigt sein, auch in diesen Löchern resp. Schalen dasselbe zu erblicken. Es scheint. mir auch, daf wir in den vielen Schalen, welche an einigen der Grabbauten in Petra angebracht sind, dasselbe Symbol vor uns haben, wie es dargestellt wird durch die Schalen auf Malsteinen und an vertikalen Wänden. Wie der Malstein-Phallus und die Vereinigung der beiden

I op. cit. p. 107. 2 Palestine Excavation Vol. I of the PEF p. 195.

3 Perrot and Chiplez, Phoenicia, engl. Übersetzung p. 287. 
Symbole bestimmte Stufen der sozialen Entwickiung dartun, so weist auch das weibliche Symbol für sich allein gleichfalls auf eine bestimmte Stufe sozialer Entwicklung. Diese Stufe kann im Hinblick auf das Symbol nur das Matriarchat sein, ein längst allgemein anerkannter $\mathrm{Zu}$ stand der menschlichen Gesellschaft in frühester Zeit. Dieser Zustand ist älter als das Patriarchat, das wird dadurch bewiesen, daß die älteste semitische Gottheit die große Gottesmutter ${ }^{x}$ war und die männliche Gottheit ihr Sohn, weicher in jener matriarchalischen Gemeinschaft der Haupt-Mann war, wie z. B. noch in Petra ${ }^{2}$ und Mekka. ${ }^{3}$. Wir finden eine weitere Bestätigung hierfür in der Tatsache, daß bei den Minäern alle männlichen Glieder der Familie als 'a $\mathrm{mm}^{4}$ bezeichnet wurden und als nächster Angehöriger nicht der Vater angesehen wurde, sondern der Onkel, wodurch sich der Einfluß des Onkels in dem späteren Arabertum bis auf den heutigen Tag erklärt. Die himmlischen Verhältnisse sind natürlich nichts anderes als ein Reflex der irdischen. Das Wort עat sich auch als alter Gottesnamen erhalten ${ }^{5}$ und WINCKLER ${ }^{6}$ glaubt, diesen Namen im AT nachweisen zu können. So liest er in Din 3242 an beiden Stellen עם "am statt עמו עמ ,sein Volk" und betrachtet vv. 36 a $\beta$ bis $38 \mathrm{a} \alpha$ als späteren Zusatz. Das Matriarchat mit dem himmlischen 'a mm als Gottheit und mit dem irdischen 'amm als das Haupt der Gemeinschaft; schaffte solche Eigennamen wie عمخراً, عمنخر ,عمكرب , عمدق ع usw.7 Der Begleiter oder Gatte der Göttin gehört einer späteren Entwicklungsstufe an, dem Patriarchat.

Im AT finden wir zwei klare Bezugnahmen auf das Matriarchat. Gemäb Gen 224 folgt der Mann dem Weibe, ein Zustand, bedingt in einer Gemeinschaft, in welcher das Matriarchat herrscht, anstatt wie es später üblich war, daß das Weib dem Manne folgte, um in dessen Familie aufgenommen zu werden. ${ }^{8}$ Die Stelle Gen 4 I hat Bezug auf dieselben sozialen Verhältnisse wie Gen 224. Das Kind gehört der Mutter in dem Matriarchat und wird ein Glied, wie auch der wahre Erhalter, des Stammes seiner Mutter. 9

Die Bitte, die Noomi an ihre beiden Schwiegertöchter richtet, Rt I 8, in die Häuser ihrer Mutter zurückzukehren, weist vielleicht auf einen ähnlichen sozialen Zustand hin wie Gen 224 und 4 r, denn gemäß späteren

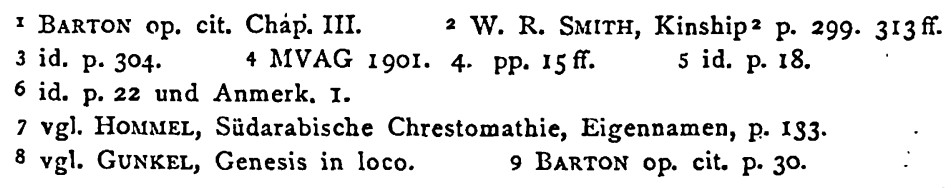


jüdischen Ansichten würde man erwarten, daß Noomi ihren Schwiegertöchtern anriete, in die Häuser ihrer Väter zurückzukehren. Dieser Teil der Erzählung mag daher sehr alte Reminiszenzen enthalten.

Als merkwürdige Nachklänge des Matriarchats, wozu wir vielleicht auch den Dienst der Himmelskönigin in gewissen Zweigen der christlichen Kirche rechnen dürfen, mögen wir gewisse Gebräuche unter den heutigen palästinensischen Juden betrachten. So wird z. B. das Kind nicht als Kind des Vaters, sondern als Kind der Mutter bezeichnet. In folgendem Gebete, welches in einer Ritze in dem Steine des Grabes Zachariae im Kidrontal, des Fürbitters der heutigen orthodoxen Juden, niedergelegt war, wird nur die Mutter, aber niemals der Vater erwähnt.

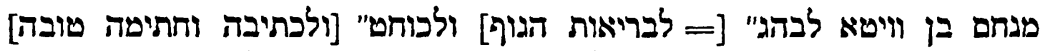

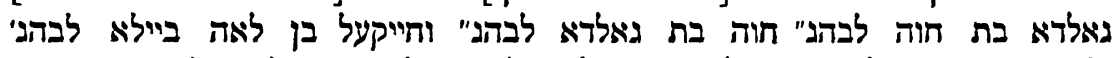

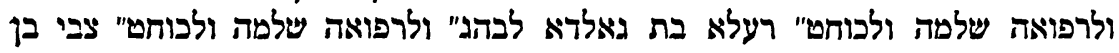

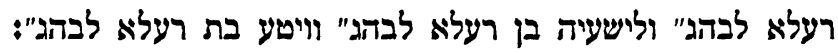

Menachem, Sohn der Wita, für Gesundheit des Körpers und für Eintragung (nämlich: seines Namens in das Buch Gottes) und ein gutes Siegel; Golda, Tochter der Hawah, für Gesundheit des Körpers; Hawah, Tochter der Goida, für Gesundheit des Körpers, und Heiq'el, Sohn der Lea Bella, für Gesundheit des Körpers und für vollständige Genesung und für Eintragung und ein gutes Siegel; Ráala, Tochter der Golda, für Gesundheit des Körpers und für vollständige Genesung und für Eintragung und ein gutes Siegel; Zebi, Sohn der Ráala, für Gesundheit des Körpers und für Ješaiah, Sohn der Racala, für Gesundheit des Körpers, Wita, Tochter der Ra'ala, für Gesundheit des Körpers [bete ich]. ${ }^{x}$

Zum Schlusse noch ein paar Worte in betreff der Schalen auf der Bama zu Gezer.?. Diese befinden sich ungefähr $40 \mathrm{~m}$ südlich von den Malsteinen, von welchen zwei oben erwähnt wurden. Die Schalen, 83 an der Zahl, sind über eine Fläche von $29 \mathrm{~m} \times 26 \mathrm{~m}$ verteilt. Sie waren verdeckt dürch drei übereinander liegende Schichten Mauerwerk, welches ebensovielen Perioden angehörte und Scherben enthielt, die nach MACALISTERs Angabe von den Höhlenbewohnern herstammten. Auf den ersten Blick möchte man wohl geneigt sein, diese Schalen mit einem Altare in Verbindung zu bringen, jedoch ihre weite Ausbreitung spricht dagegen. In der Nähe dieser Schalen befinden sich Malsteine, die ebenfalls der ältesten Periode angehören; das Ganze gleicht der Gruppe von Malsteinen

I Dieses Gebet ist augenscheinlich ein Neujahrsgebet, da an diesem Tage, nach dem Glauben der orthodoxen Juden, Got: die Geschicke der Menschen bis ins kleinste hinein anordnet. 2 Quart. Stat. PEF. 1903 pp. 317 ff.

Zeitschrift f. d. alttest. Wiss: Jahrg. 28. 1908. 
zu El-mareiğât, welche einen Kreis von ungefähr $270 \mathrm{~m}$ im Durchmesser bildet. ${ }^{x}$ Die Steine zu Mareiğât sind Kultsymbole und so liegt der Gedanke nahe, dab die Schalen zu Gezer auch solche sind. Diese Annahme findet eine Bekräftigung in den Malsteinen, deren Charakter oben erklärt worden ist. Ich fühle mich daher auch veranlabt, diese Schalensteine als Symbole der weiblichen Gottheit anzusehen. Dasselbe trifft denn auch im allgemeinen zu für die Gruppen von Schalen, die man an anderen Orten findet, z. B. in der Nähe von Silwân, Dilb, Áin ğidi. An letzterem Orte findet sich an der Quelle ein sehr großer Felsblock, der viele Schalen aufweist, an der Seite sowohl wie oben; für andere Orte vergleiche man den Survey of Palestine.

Die große Verbreitung dieses Kultes in Gezer ist bestätigt durch die vielen Funde von Astartecippi ${ }^{2}$ und Phalli, ${ }^{3}$ jedoch gehören diese Kultobjekte wohl einer späteren Zeit an als die roheren und größeren Darstellungen derselben religiösen Ideen, wie wir sie symbolisiert finden in den Schalen- und Malsteinen.

I Survey pp. $186 \mathrm{f}$.

2 Report on the Excavation of Gezer by R. A. S. Macalister p. 89

3 id. p. 26. 